\title{
Meromorphic functions sharing fixed points and poles with finite weights
}

\author{
Pulak Sahoo \\ University of Kalyani \\ Department of Mathematics \\ West Bengal 741235, India \\ email: sahoopulak1@gmail.com
}

\begin{abstract}
In the paper, with the aid of weighted sharing method we study the problems of meromorphic functions that share fixed points (or a nonzero finite value) and poles with finite weights. The results of the paper improve some recent results due to Y. H. Cao and X. B. Zhang [Journal of Inequalities and Applications, 2012:100].
\end{abstract}

\section{Introduction, definitions and results}

In this paper, by meromorphic functions we will always mean meromorphic functions in the complex plane. We adopt the standard notations in the Nevanlinna theory of meromorphic functions as explained in [8], [15] and [16]. For a nonconstant meromorphic function $f$, we denote by $T(r, f)$ the Nevanlinna characteristic of $f$ and by $S(r, f)$ any quantity satisfying $S(r, f)=o\{T(r, f)\}$ as $r \rightarrow \infty$ possibly outside a set of finite linear measure. A meromorphic function $\alpha(z)(\not \equiv \infty)$ is called a small function with respect to $f$, provided that $\mathrm{T}(\mathrm{r}, \alpha)=\mathrm{S}(\mathrm{r}, \mathrm{f})$.

We say that two meromorphic functions $f$ and $g$ share a small function $a(z) C M$, provided that $f-a$ and $g-a$ have the same zeros with the same multiplicities. Similarly, we say that $f$ and $g$ share $a(z)$ IM, provided that $f-a$ and $g-a$ have the same zeros ignoring multiplicities. In addition, we say that $f$ and $g$ share $\infty \mathrm{CM}$, if $\frac{1}{f}$ and $\frac{1}{g}$ share $0 \mathrm{CM}$, and we say that $f$ and $g$ share $\infty$ 
IM, if $\frac{1}{f}$ and $\frac{1}{g}$ share 0 IM. A finite value $z_{0}$ is a fixed point of $f(z)$ if $f\left(z_{0}\right)=z_{0}$ and we define

$$
\mathrm{E}_{f}=\{z \in \mathbb{C}: f(z)=z \text {, counting multiplicities }\} .
$$

In 1995, W. Bergweiler and A. Eremenko, H. H. Chen and M. L. Fang, L. Zalcman respectively proved the following result.

Theorem A (see ([3], Theorem 2), ([5], Theorem 1) and [17]) Let $\mathrm{f}$ be a transcendental meromorphic function and $\mathrm{n}(\geq 1)$ is an integer. Then $\mathrm{f}^{\mathrm{n}} \mathrm{f}^{\prime}=1$ has infinitely many solutions.

In 1997, C. C. Yang and X. H. Hua proved the following result, which corresponded to Theorem A.

Theorem B (see [14], Theorem 1) Let $\mathrm{f}$ and $\mathrm{g}$ be two nonconstant meromorphic functions, $\mathrm{n} \geq 11$ be a positive integer. If $\mathrm{f}^{n} \mathrm{f}^{\prime}$ and $\mathrm{g}^{\mathrm{n}} \mathrm{g}^{\prime}$ share $1 C M$, then either $\mathrm{f}(z)=\mathrm{c}_{1} \mathrm{e}^{\mathrm{cz}}, \mathrm{g}(z)=\mathrm{c}_{2} \mathrm{e}^{-\mathrm{c} z}$, where $\mathrm{c}_{1}, \mathrm{c}_{2}$ and $\mathrm{c}$ are three constants satisfying $\left(\mathrm{c}_{1} \mathrm{c}_{2}\right)^{\mathrm{n}+1} \mathrm{c}^{2}=-1$ or $\mathrm{f} \equiv \mathrm{tg}$ for a constant $\mathrm{t}$ such that $\mathrm{t}^{\mathrm{n}+1}=1$.

In 2000, M. L. Fang proved the following result.

Theorem C (see [6], Theorem 2) Let $\mathrm{f}$ be a transcendental meromorphic function, and let $\mathrm{n}$ be a positive integer. Then $\mathrm{f}^{n} \mathrm{f}^{\prime}-z=0$ has infinitely many solutions.

In 2002, M. L. Fang and H. L. Qiu proved the following result, which corresponded to Theorem $\mathrm{C}$.

Theorem D (see [7], Theorem 1) Let $\mathrm{f}$ and $\mathrm{g}$ be two nonconstant meromorphic functions, and let $\mathrm{n} \geq 11$ be a positive integer. If $\mathrm{f}^{\mathrm{n}} \mathrm{f}^{\prime}-z$ and $\mathrm{g}^{\mathrm{n}} \mathrm{g}^{\prime}-z$ share $0 C M$, then either $\mathrm{f}(z)=\mathrm{c}_{1} e^{\mathrm{c} z^{2}}, \mathrm{~g}(z)=\mathrm{c}_{2} e^{-\mathrm{c} z^{2}}$, where $\mathrm{c}_{1}, \mathrm{c}_{2}$ and $\mathrm{c}$ are three nonzero complex numbers satisfying $4\left(\mathrm{c}_{1} \mathrm{c}_{2}\right)^{\mathrm{n}+1} \mathrm{c}^{2}=-1$ or $\mathrm{f}=\operatorname{tg}$ for a complex number $\mathrm{t}$ such that $\mathrm{t}^{\mathrm{n}+1}=1$.

In 2009, J. F. Xu, H. X. Yi and Z. L. Zhang proved the following result.

Theorem $\mathbf{E}$ (see [12]) Let $\mathrm{f}$ be a transcendental meromorphic function, $\mathrm{n}(\geq$ $2), \mathrm{k}$ be two positive integers. Then $\mathrm{f}^{\mathrm{n}} \mathrm{f}^{(\mathrm{k})}$ takes every finite nonzero value infinitely many times or has infinitely many fixed points.

Regarding Theorem E, it is natural to ask the following question:

Question 1 Is there a corresponding uniqueness theorem to Theorem E? 
Recently, Y. H. Cao and X. B. Zhang proved the following results which deal with Question 1.

Theorem F (see [4], Theorem 1.1) Let $\mathrm{f}$ and $\mathrm{g}$ be two transcendental meromorphic functions, whose zeros are of multiplicities at least $\mathrm{k}$, where $\mathrm{k}$ is a positive integer. Let $\mathrm{n}>\max \{2 \mathrm{k}-1, \mathrm{k}+4 / \mathrm{k}+4\}$ be a positive integer. If $\mathrm{f}^{\mathrm{n}} \mathrm{f}^{(\mathrm{k})}$ and $\mathrm{g}^{\mathrm{n}} \mathrm{g}^{(\mathrm{k})}$ share $\mathrm{z} C M, \mathrm{f}$ and $\mathrm{g}$ share $\infty \mathrm{IM}$, then one of the following two conclusions hold:

(i) $f^{n} f^{(k)}=g^{n} g^{(k)}$;

(ii) $\mathrm{f}(z)=\mathrm{c}_{1} e^{\mathrm{cz^{2 }}}, \mathrm{g}(z)=\mathrm{c}_{2} e^{-\mathrm{c} z^{2}}$, where $\mathrm{c}_{1}, \mathrm{c}_{2}$ and $\mathrm{c}$ are constants satisfying $4\left(c_{1} c_{2}\right)^{n+1} c^{2}=-1$.

Theorem G (see [4], Theorem 1.2) Let $\mathrm{f}$ and $\mathrm{g}$ be two nonconstant meromorphic functions, whose zeros are of multiplicities at least $\mathrm{k}$, where $\mathrm{k}$ is a positive integer. Let $\mathrm{n}>\max \{2 \mathrm{k}-1, \mathrm{k}+4 / \mathrm{k}+4\}$ be a positive integer. If $\mathrm{f}^{\mathrm{n}} \mathrm{f}^{(\mathrm{k})}$ and $\mathrm{g}^{\mathrm{n}} \mathrm{g}^{(\mathrm{k})}$ share $1 C M, \mathrm{f}$ and $\mathrm{g}$ share $\infty I M$, then one of the following two conclusions hold:

(i) $f^{n} f^{(k)}=g^{n} g^{(k)}$;

(ii) $\mathrm{f}(z)=\mathrm{c}_{3} \mathrm{e}^{\mathrm{d} z}, \mathrm{~g}(z)=\mathrm{c}_{4} \mathrm{e}^{-\mathrm{d} z}$, where $\mathrm{c}_{3}, \mathrm{c}_{4}$ and $\mathrm{d}$ are constants satisfying $(-1)^{k}\left(c_{3} c_{4}\right)^{n+1} d^{2 k}=1$.

Regarding Theorem F and Theorem G, one may ask the following questions which are the motive of the author.

Question 2 Is it really possible in any way to relax the nature of sharing the fixed point (1-point) in Theorem F (Theorem G) without increasing the lower bound of $n$ ?

Question 3 What will be the IM-analogous of Theorems $F$ and G?

In the paper, we will prove two theorems first one of which improves Theorem F and second one improves Theorem G and dealt with Question 2 and Question 3. To state the main results of the paper we need the following notion of weighted sharing of values introduced by I. Lahiri $[9,10]$ which measure how close a shared value is to being shared CM or to being shared IM.

Definition 1 Let $\mathrm{k}$ be a nonnegative integer or infinity. For $\mathrm{a} \in \mathbb{C} \cup\{\infty\}$ we denote by $\mathrm{E}_{\mathrm{k}}(\mathrm{a} ; \mathrm{f})$ the set of all $\mathrm{a}$-points of $\mathrm{f}$ where an a-point of multiplicity 
$m$ is counted $m$ times if $\mathrm{m} \leq \mathrm{k}$ and $k+1$ times if $\mathrm{m}>\mathrm{k}$. If $\mathrm{E}_{\mathrm{k}}(\mathrm{a} ; \mathrm{f})=\mathrm{E}_{\mathrm{k}}(\mathrm{a} ; \mathrm{g})$, we say that $\mathrm{f}, \mathrm{g}$ share the value a with weight $k$.

The definition implies that if $\mathrm{f}, \mathrm{g}$ share a value a with weight $\mathrm{k}$, then $z_{0}$ is an a-point of $\mathrm{f}$ with multiplicity $\mathrm{m}(\leq \mathrm{k})$ if and only if it is an a-point of $\mathrm{g}$ with multiplicity $\mathrm{m}(\leq \mathrm{k})$ and $z_{0}$ is an a-point of $\mathrm{f}$ with multiplicity $\mathrm{m}(>\mathrm{k})$ if and only if it is an a-point of $\mathrm{g}$ with multiplicity $\mathrm{n}(>\mathrm{k})$, where $m$ is not necessarily equal to $n$.

We write $\mathrm{f}, \mathrm{g}$ share $(\mathrm{a}, \mathrm{k})$ to mean that $\mathrm{f}, \mathrm{g}$ share the value a with weight $k$. Clearly if $\mathrm{f}, \mathrm{g}$ share $(\mathrm{a}, \mathrm{k})$ then $\mathrm{f}, \mathrm{g}$ share $(\mathrm{a}, \mathrm{p})$ for any integer $\mathrm{p}, 0 \leq \mathrm{p}<\mathrm{k}$. Also we note that $\mathrm{f}, \mathrm{g}$ share a value a IM or CM if and only if $\mathrm{f}, \mathrm{g}$ share $(a, 0)$ or $(a, \infty)$ respectively.

We now state the main results of the paper.

Theorem 1 Let $\mathrm{f}$ and $\mathrm{g}$ be two transcendental meromorphic functions, whose zeros are of multiplicities at least $\mathrm{k}$, where $\mathrm{k}$ is a positive integer. If $\mathrm{f}^{\mathrm{n}} \mathrm{f}^{(\mathrm{k})}$ and $\mathrm{g}^{\mathrm{n}} \mathrm{g}^{(\mathrm{k})}$ share $(\mathrm{z}, \mathrm{l})$, where $\mathrm{l}, \mathrm{n}$ are positive integers; $\mathrm{f}$ and $\mathrm{g}$ share $\infty I M$, then conclusions of Theorem $F$ hold provided one of the following holds:

(i) $l \geq 2$ and $n>\max \{2 k-1, k+4 / k+4\}$;

(ii) $l=1$ and $n>\max \{2 k-1,3 k / 2+5 / k+5\}$;

(iii) $l=0$ and $n>\max \{2 k-1,4 k+10 / k+10\}$.

Theorem 2 Let $\mathrm{f}$ and $\mathrm{g}$ be two nonconstant meromorphic functions, whose zeros are of multiplicities at least $\mathrm{k}$, where $\mathrm{k}$ is a positive integer. If $\mathrm{f}^{\mathrm{n}} \mathrm{f}^{(\mathrm{k})}$ and $\mathrm{g}^{\mathrm{n}} \mathrm{g}^{(\mathrm{k})}$ share $(1, \mathrm{l})$, where $\mathrm{l}, \mathrm{n}$ are positive integers; $\mathrm{f}$ and $\mathrm{g}$ share $\infty I M$, then conclusions of Theorem $G$ hold provided one of the following holds:

(i) $l \geq 2$ and $n>\max \{2 k-1, k+4 / k+4\}$;

(ii) $l=1$ and $n>\max \{2 k-1,3 k / 2+5 / k+5\}$;

(iii) $l=0$ and $n>\max \{2 k-1,4 k+10 / k+10\}$.

We now explain some definitions and notations which are used in the paper.

Definition $2[8]$ For $a \in \mathbb{C} \cup\{\infty\}$ we denote by $\mathrm{N}(\mathrm{r}, \mathrm{a} ; \mathrm{f} \mid=1)$ the counting functions of simple a-points of $\mathrm{f}$. For a positive integer $\mathrm{p}$ we denote by $\mathrm{N}(\mathrm{r}, \mathrm{a} ; \mathrm{f} \mid \geq p)$ the counting function of those a-points of $\mathrm{f}$ (counted with proper multiplicities) whose multiplicities are not less than $p . B y \bar{N}(r, a ; f \mid \geq p)$ we denote the corresponding reduced counting function.

Analogously we can define $\mathrm{N}(\mathrm{r}, \mathrm{a} ; \mathrm{f} \mid \leq \mathrm{p})$ and $\overline{\mathrm{N}}(\mathrm{r}, \mathrm{a} ; \mathrm{f} \mid \leq \mathrm{p})$. 
Definition 3 [10] Let $\mathrm{k}$ be a positive integer or infinity. We denote by $\mathrm{N}_{\mathrm{k}}(\mathrm{r}, \mathrm{a} ; \mathrm{f})$ the counting function of a-points of $\mathrm{f}$, where an a-point of multiplicity $\mathrm{m}$ is counted $\mathrm{m}$ times if $\mathrm{m} \leq \mathrm{k}$ and $\mathrm{k}$ times if $\mathrm{m}>\mathrm{k}$. Then

$$
N_{k}(r, a ; f)=\bar{N}(r, a ; f)+\bar{N}(r, a ; f \mid \geq 2)+\cdots+\bar{N}(r, a ; f \mid \geq k) .
$$

Clearly $\mathrm{N}_{1}(\mathrm{r}, \mathrm{a} ; \mathrm{f})=\overline{\mathrm{N}}(\mathrm{r}, \mathrm{a} ; \mathrm{f})$.

Definition 4 [1] Let $\mathrm{f}$ and $\mathrm{g}$ be two nonconstant meromorphic functions such that $\mathrm{f}$ and $\mathrm{g}$ share the value $1 \mathrm{IM}$. Let $\mathrm{z}_{0}$ be a 1-point of $\mathrm{f}$ with multiplicity $\mathrm{p}$ and also a 1-point of $\mathrm{g}$ with multiplicity $\mathrm{q}$. We denote by $\overline{\mathrm{N}}_{\mathrm{L}}(\mathrm{r}, 1 ; \mathrm{f})$ the counting function of those 1 -points of $\mathrm{f}$ and $\mathrm{g}$, where $\mathrm{p}>\mathrm{q}$, by $\mathrm{N}_{\mathrm{E}}^{(\mathrm{k}}(\mathrm{r}, 1 ; \mathrm{f})$ $(\mathrm{k} \geq 2$ is an integer) the counting function of those 1-points of $\mathrm{f}$ and $\mathrm{g}$, where $\mathrm{p}=\mathrm{q} \geq \mathrm{k}$, where each point in these counting functions is counted only once. In the same manner we can define $\overline{\mathrm{N}}_{\mathrm{L}}(\mathrm{r}, 1 ; \mathrm{g})$ and $\mathrm{N}_{\mathrm{E}}^{(\mathrm{k}}(\mathrm{r}, 1 ; \mathrm{g})$.

Definition $\mathbf{5}[9,10]$ Let $\mathrm{f}$ and $\mathrm{g}$ be two nonconstant meromorphic functions such that $\mathrm{f}$ and $\mathrm{g}$ share the value a $I M$. We denote by $\overline{\mathrm{N}}_{*}(\mathrm{r}, \mathrm{a} ; \mathrm{f}, \mathrm{g})$ the reduced counting function of those a-points of $\mathrm{f}$ whose multiplicities differ from the multiplicities of the corresponding a-points of g. Clearly $\overline{\mathrm{N}}_{*}(\mathrm{r}, \mathrm{a} ; \mathrm{f}, \mathrm{g})=$ $\bar{N}_{*}(r, a ; g, f)$ and $\bar{N}_{*}(r, a ; f, g)=\bar{N}_{L}(r, a ; f)+\bar{N}_{L}(r, a ; g)$.

\section{Lemmas}

In this section we present some lemmas which will be needed in the sequel. Let $F$ and $G$ be two nonconstant meromorphic functions defined in $\mathbb{C}$. We shall denote by $\mathrm{H}$ the following function:

$$
H=\left(\frac{F^{\prime \prime}}{F^{\prime}}-\frac{2 F^{\prime}}{F-1}\right)-\left(\frac{G^{\prime \prime}}{G^{\prime}}-\frac{2 G^{\prime}}{G-1}\right) .
$$

Lemma 1 [13] Let $\mathrm{f}$ be a nonconstant meromorphic function and let $\mathrm{a}_{\mathfrak{n}}(z)(\not \equiv$ $0), a_{n-1}(z), \ldots, a_{0}(z)$ be meromorphic functions such that $\mathrm{T}\left(\mathrm{r}, \mathrm{a}_{\mathrm{i}}(z)\right)=\mathrm{S}(\mathrm{r}, \mathrm{f})$ for $i=0,1,2, \ldots, n$. Then

$$
T\left(r, a_{n} f^{n}+a_{n-1} f^{n-1}+\ldots+a_{1} f+a_{0}\right)=n T(r, f)+S(r, f) .
$$

Lemma 2 [16] Let $\mathrm{f}$ be a nonconstant meromorphic function, and let $\mathrm{k}$ be positive integer. Suppose that $\mathrm{f}^{(\mathrm{k})} \not \equiv 0$. Then

$$
N\left(r, 0 ; f^{(k)}\right) \leq N(r, 0 ; f)+k \bar{N}(r, \infty ; f)+S(r, f) .
$$


Lemma 3 [18] Let $\mathrm{f}$ be a nonconstant meromorphic function, and $\mathrm{p}, \mathrm{k}$ be positive integers. Then

$$
N_{p}\left(r, 0 ; f^{(k)}\right) \leq k \bar{N}(r, \infty ; f)+N_{p+k}(r, 0 ; f)+S(r, f) .
$$

Lemma 4 [2] Let $\mathrm{F}, \mathrm{G}$ be two nonconstant meromorphic functions sharing $(1,2),(\infty, 0)$ and $\mathrm{H} \not \equiv 0$. Then

(i) $\mathrm{T}(\mathrm{r}, \mathrm{F}) \leq \mathrm{N}_{2}(\mathrm{r}, 0 ; \mathrm{F})+\mathrm{N}_{2}(\mathrm{r}, 0 ; \mathrm{G})+\overline{\mathrm{N}}(\mathrm{r}, \infty ; \mathrm{F})+\overline{\mathrm{N}}(\mathrm{r}, \infty ; \mathrm{G})+\overline{\mathrm{N}}_{*}(\mathrm{r}, \infty ; \mathrm{F}, \mathrm{G})-$ $m(r, 1 ; G)-N_{E}^{(3}(r, 1 ; F)-\bar{N}_{L}(r, 1 ; G)+S(r, F)+S(r, G) ;$

(ii) $\mathrm{T}(r, \mathrm{G}) \leq \mathrm{N}_{2}(r, 0 ; F)+\mathrm{N}_{2}(r, 0 ; G)+\overline{\mathrm{N}}(r, \infty ; F)+\overline{\mathrm{N}}(r, \infty ; G)+\overline{\mathrm{N}}_{*}(r, \infty ; F, G)-$ $m(r, 1 ; F)-N_{E}^{(3}(r, 1 ; G)-\bar{N}_{L}(r, 1 ; F)+S(r, F)+S(r, G)$.

Lemma 5 [11] Let $\mathrm{F}, \mathrm{G}$ be two nonconstant meromorphic functions sharing $(1,1),(\infty, 0)$ and $\mathrm{H} \not \equiv 0$. Then

(i) $\mathrm{T}(r, F) \leq \mathrm{N}_{2}(r, 0 ; F)+\mathrm{N}_{2}(r, 0 ; G)+\frac{3}{2} \bar{N}(r, \infty ; F)+\bar{N}(r, \infty ; G)+\frac{1}{2} \bar{N}(r, 0 ; F)+$ $\bar{N}_{*}(r, \infty ; F, G)+S(r, F)+S(r, G)$

(ii) $T(r, G) \leq N_{2}(r, 0 ; F)+N_{2}(r, 0 ; G)+\bar{N}(r, \infty ; F)+\frac{3}{2} \bar{N}(r, \infty ; G)+\frac{1}{2} \bar{N}(r, 0 ; G)+$ $\bar{N}_{*}(r, \infty ; F, G)+S(r, F)+S(r, G)$.

Lemma 6 [11] Let $\mathrm{F}, \mathrm{G}$ be two nonconstant meromorphic functions sharing $(1,0),(\infty, 0)$ and $\mathrm{H} \not \equiv 0$. Then

(i) $\mathrm{T}(\mathrm{r}, \mathrm{F}) \leq \mathrm{N}_{2}(r, 0 ; F)+\mathrm{N}_{2}(r, 0 ; G)+3 \overline{\mathrm{N}}(\mathrm{r}, \infty ; \mathrm{F})+2 \overline{\mathrm{N}}(\mathrm{r}, \infty ; \mathrm{G})+2 \overline{\mathrm{N}}(r, 0 ; \mathrm{F})+$ $\bar{N}(r, 0 ; G)+\bar{N}_{*}(r, \infty ; F, G)+S(r, F)+S(r, G)$;

(ii) $\mathrm{T}(\mathrm{r}, \mathrm{G}) \leq \mathrm{N}_{2}(\mathrm{r}, 0 ; \mathrm{F})+\mathrm{N}_{2}(\mathrm{r}, 0 ; \mathrm{G})+2 \overline{\mathrm{N}}(\mathrm{r}, \infty ; \mathrm{F})+3 \overline{\mathrm{N}}(\mathrm{r}, \infty ; \mathrm{G})+\overline{\mathrm{N}}(\mathrm{r}, 0 ; \mathrm{F})+$ $2 \bar{N}(r, 0 ; G)+\bar{N}_{*}(r, \infty ; F, G)+S(r, F)+S(r, G)$.

Lemma 7 [4] Let $\mathrm{f}$ and $\mathrm{g}$ be nonconstant meromorphic functions, whose zeros are of multiplicities at least $\mathrm{k}$, where $\mathrm{k}$ is a positive integer. Let $\mathrm{n}>2 \mathrm{k}-$ 1 be a positive integer. If $\mathrm{f}, \mathrm{g}$ share $\infty$ IM and if $\mathrm{f}^{\mathrm{n}} \mathrm{f}^{(\mathrm{k})} \mathrm{g}^{\mathrm{n}} \mathrm{g}^{(\mathrm{k})}=z^{2}$, then $\mathrm{f}(z)=\mathrm{c}_{1} e^{\mathrm{c} z^{2}}, \mathrm{~g}(z)=\mathrm{c}_{2} e^{-\mathrm{c} z^{2}}$, where $\mathrm{c}_{1}, \mathrm{c}_{2}$ and $\mathrm{c}$ are three constants satisfying $4\left(c_{1} c_{2}\right)^{n+1} c^{2}=-1$.

Lemma 8 [4] Let $\mathrm{f}$ and $\mathrm{g}$ be nonconstant meromorphic functions, whose zeros are of multiplicities at least $\mathrm{k}$, where $\mathrm{k}$ is a positive integer. Let $\mathrm{n}>2 \mathrm{k}-$ 1 be a positive integer. If $\mathrm{f}, \mathrm{g}$ share $\infty I M$ and if $\mathrm{f}^{\mathrm{n}} \mathrm{f}^{(\mathrm{k})} \mathrm{g}^{\mathrm{n}} \mathrm{g}^{(\mathrm{k})}=1$, then $\mathrm{f}(z)=\mathrm{c}_{3} \mathrm{e}^{\mathrm{d} z}, \mathrm{~g}(z)=\mathrm{c}_{4} \mathrm{e}^{-\mathrm{d} z}$, where $\mathrm{c}_{3}, \mathrm{c}_{4}$ and $\mathrm{d}$ are three constants satisfying $(-1)^{k}\left(c_{3} c_{4}\right)^{n+1} d^{2 k}=1$. 


\section{Proof of the theorems}

Proof of Theorem 1. We consider $F(z)=f^{n} f^{(k)}, G(z)=g^{n} g^{(k)}, F_{1}(z)=$ $\mathrm{F}(z) / z$ and $\mathrm{G}_{1}(z)=\mathrm{G}(z) / z$. Then $\mathrm{F}_{1}, \mathrm{G}_{1}$ are transcendental meromorphic functions that share $(1, l)$ and $f, g$ share $(\infty, 0)$. Since $f$ and $g$ are transcendental, $z$ is a small function with respect to both $F$ and $G$. We now discuss the following two cases separately.

Case 1 We assume that $\mathrm{H} \not \equiv 0$. Now we consider the following three subcases.

Subcase 1 Suppose that $\mathrm{l} \geq 2$. Then using Lemma \& we obtain

$$
\begin{aligned}
T(r, F) \leq & T\left(r, F_{1}\right)+S(r, F) \\
\leq & N_{2}\left(r, 0 ; F_{1}\right)+N_{2}\left(r, 0 ; G_{1}\right)+\bar{N}\left(r, \infty ; F_{1}\right)+\bar{N}\left(r, \infty ; G_{1}\right) \\
& +\bar{N}_{*}\left(r, \infty ; F_{1}, G_{1}\right)-m\left(r, 1 ; G_{1}\right)-N_{E}^{(3}\left(r, 1 ; F_{1}\right) \\
& -\bar{N}_{L}\left(r, 1 ; G_{1}\right)+S\left(r, F_{1}\right)+S\left(r, G_{1}\right) \\
\leq & N_{2}(r, 0 ; F)+N_{2}(r, 0 ; G)+\bar{N}(r, \infty ; F)+\bar{N}(r, \infty ; G) \\
& +\bar{N}_{*}(r, \infty ; F, G)+S(r, F)+S(r, G) .
\end{aligned}
$$

Noting that

$$
\begin{aligned}
\bar{N}_{*}(r, \infty ; F, G) & =\bar{N}_{L}(r, \infty ; F)+\bar{N}_{L}(r, \infty ; G) \\
& \leq \bar{N}(r, \infty ; F)=\bar{N}(r, \infty ; G),
\end{aligned}
$$

we obtain from (3) that

$$
\begin{aligned}
T(r, F) \leq & N_{2}(r, 0 ; F)+N_{2}(r, 0 ; G)+2 \bar{N}(r, \infty ; F)+\bar{N}(r, \infty ; G) \\
& +S(r, F)+S(r, G) .
\end{aligned}
$$

Obviously,

$$
N(r, \infty ; F)=(n+1) N(r, \infty ; f)+k \bar{N}(r, \infty ; f)+S(r, f)
$$

Again

$$
\begin{aligned}
n m(r, f) & =m\left(r, F / f^{(k)}\right) \leq m(r, F)+m\left(r, 1 / f^{(k)}\right)+S(r, f) \\
& =m(r, F)+T\left(r, f^{(k)}\right)-N\left(r, 0 ; f^{(k)}\right)+S(r, f) \\
& \leq m(r, F)+T(r, f)+k \bar{N}(r, \infty ; f)-N\left(r, 0 ; f^{(k)}\right)+S(r, f) .
\end{aligned}
$$


From (6) and (7) we obtain

$$
(n-1) T(r, f) \leq T(r, F)-N(r, \infty ; f)-N\left(r, 0 ; f^{(k)}\right)+S(r, f) .
$$

Similarly,

$$
(n-1) T(r, g) \leq T(r, G)-N(r, \infty ; g)-N\left(r, 0 ; g^{(k)}\right)+S(r, g) .
$$

Using (6), Lemma 2 we obtain from (8)

$$
\begin{aligned}
(n-1) T(r, f) \leq & N_{2}(r, 0 ; F)+N_{2}(r, 0 ; G)+2 \bar{N}(r, \infty ; F)+\bar{N}(r, \infty ; G) \\
& -N(r, \infty ; f)-N\left(r, 0 ; f^{(k)}\right)+S(r, f)+S(r, g) \\
\leq & N_{2}(r, 0 ; f)+N_{2}(r, 0 ; g)+N_{2}\left(r, 0 ; f^{(k)}\right)+N_{2}\left(r, 0 ; g^{(k)}\right) \\
& +2 \bar{N}(r, \infty ; f)+\bar{N}(r, \infty ; g)-N(r, \infty ; f) \\
& -N\left(r, 0 ; f^{(k)}\right)+S(r, f)+S(r, g) \\
\leq & 2 \bar{N}(r, 0 ; f)+2 \bar{N}(r, 0 ; g)+N\left(r, 0 ; f^{(k)}\right)+N\left(r, 0 ; g^{(k)}\right) \\
& +2 N(r, \infty ; f)+\bar{N}(r, \infty ; g)-N(r, \infty ; f) \\
& -N\left(r, 0 ; f^{(k)}\right)+S(r, f)+S(r, g) \\
\leq & 2 \bar{N}(r, 0 ; f)+2 \bar{N}(r, 0 ; g)+N(r, 0 ; g)+N(r, \infty ; f) \\
& +(k+1) \bar{N}(r, \infty ; g)+S(r, f)+S(r, g) \\
\leq & \frac{2}{k} N(r, 0 ; f)+\frac{2}{k} N(r, 0 ; g)+N(r, 0 ; g)+N(r, \infty ; g) \\
& +(k+1) \bar{N}(r, \infty ; g)+S(r, f)+S(r, g) \\
\leq & \frac{2}{k}(T(r, f)+T(r, g))+(k+3) T(r, g) \\
& +S(r, f)+S(r, g) .
\end{aligned}
$$

Similarly,

$$
\begin{aligned}
(n-1) T(r, g) \leq & \frac{2}{k}(T(r, f)+T(r, g))+(k+3) T(r, f) \\
& +S(r, f)+S(r, g) .
\end{aligned}
$$

Combining (10) and (11) we get

$$
(n-k-4 / k-4)(T(r, f)+T(r, g)) \leq S(r, f)+S(r, g),
$$

a contradiction with the fact that $\mathrm{n}>\mathrm{k}+4 / \mathrm{k}+4$. 
Subcase 2 Let $l=1$. Then using (4) and Lemma 5 we obtain

$$
\begin{aligned}
T(r, F) \leq & T\left(r, F_{1}\right)+S(r, F) \\
\leq & N_{2}\left(r, 0 ; F_{1}\right)+N_{2}\left(r, 0 ; G_{1}\right)+\frac{3}{2} \bar{N}\left(r, \infty ; F_{1}\right)+\bar{N}\left(r, \infty ; G_{1}\right) \\
& +\bar{N}_{*}\left(r, \infty ; F_{1}, G_{1}\right)+\frac{1}{2} \bar{N}\left(r, 0 ; F_{1}\right)+S\left(r, F_{1}\right)+S\left(r, G_{1}\right) \\
\leq & N_{2}(r, 0 ; F)+N_{2}(r, 0 ; G)+\frac{3}{2} \bar{N}(r, \infty ; F)+\bar{N}(r, \infty ; G) \\
& +\bar{N}_{*}(r, \infty ; F, G)+\frac{1}{2} \bar{N}(r, 0 ; F)+S(r, F)+S(r, G) \\
\leq & N_{2}(r, 0 ; F)+N_{2}(r, 0 ; G)+\frac{5}{2} \bar{N}(r, \infty ; F)+\bar{N}(r, \infty ; G) \\
& +\frac{1}{2} \bar{N}(r, 0 ; F)+S(r, F)+S(r, G) .
\end{aligned}
$$

Using (12), Lemma 2 and Lemma 3 we obtain from (8)

$$
\begin{aligned}
(n-1) T(r, f) \leq & N_{2}(r, 0 ; F)+N_{2}(r, 0 ; G)+\frac{5}{2} \bar{N}(r, \infty ; F)+\bar{N}(r, \infty ; G) \\
& +\frac{1}{2} \bar{N}(r, 0 ; F)-N(r, \infty ; f)-N\left(r, 0 ; f^{(k)}\right) \\
& +S(r, f)+S(r, g) \\
\leq & N_{2}(r, 0 ; f)+N_{2}(r, 0 ; g)+N_{2}\left(r, 0 ; f^{(k)}\right)+N_{2}\left(r, 0 ; g^{(k)}\right) \\
& +\frac{5}{2} \bar{N}(r, \infty ; f)+\bar{N}(r, \infty ; g)+\frac{1}{2} \bar{N}(r, 0 ; f) \\
& +\frac{1}{2} \bar{N}\left(r, 0 ; f^{(k)}\right)-N(r, \infty ; f)-N\left(r, 0 ; f^{(k)}\right) \\
& +S(r, f)+S(r, g) \\
\leq & \frac{5}{2} \bar{N}(r, 0 ; f)+2 \bar{N}(r, 0 ; g)+N\left(r, 0 ; f^{(k)}\right)+N\left(r, 0 ; g^{(k)}\right) \\
& +\frac{5}{2} N(r, \infty ; f)+\bar{N}(r, \infty ; g)+\frac{1}{2} N(k+1(r, 0 ; f) \\
& +\frac{k}{2} \bar{N}(r, \infty ; f)-N(r, \infty ; f)-N(r, 0 ; f(k)) \\
& +S(r, f)+S(r, g) \\
\leq & \frac{k+6}{2} \bar{N}(r, 0 ; f)+2 \bar{N}(r, 0 ; g)+(k+1) \bar{N}(r, \infty ; g) \\
& +N(r, 0 ; g)+\frac{k+3}{2} N(r, \infty ; f)+S(r, f)+S(r, g)
\end{aligned}
$$




$$
\begin{aligned}
\leq & \frac{k+6}{2 k} \mathrm{~N}(r, 0 ; f)+\frac{k+2}{k} \mathrm{~N}(r, 0 ; g)+\frac{3 k+5}{2} \mathrm{~N}(r, \infty ; g) \\
& +S(r, f)+S(r, g) \\
\leq & \left(\frac{2}{k}+\frac{1}{2}\right)(T(r, f)+T(r, g))+\frac{1}{k} \mathrm{~T}(r, f)+\frac{3 k+6}{2} \mathrm{~T}(r, g) \\
& +S(r, f)+S(r, g) .
\end{aligned}
$$

This implies

$$
\begin{aligned}
\left(n-1-\frac{1}{k}\right) T(r, f) & \leq\left(\frac{2}{k}+\frac{1}{2}\right)(T(r, f)+T(r, g)) \\
& +\frac{3 k+6}{2} T(r, g)+S(r, f)+S(r, g) .
\end{aligned}
$$

Similarly,

$$
\begin{aligned}
\left(n-1-\frac{1}{k}\right) T(r, g) & \leq\left(\frac{2}{k}+\frac{1}{2}\right)(T(r, f)+T(r, g)) \\
& +\frac{3 k+6}{2} T(r, f)+S(r, f)+S(r, g) .
\end{aligned}
$$

From (13) and (14) we obtain

$$
\left(n-\frac{3 k}{2}-\frac{5}{k}-5\right)(T(r, f)+T(r, g)) \leq S(r, f)+S(r, g),
$$

a contradiction with our assumption that $\mathrm{n}>3 \mathrm{k} / 2+5 / \mathrm{k}+5$.

Subcase 3 Let $l=0$. Then using (4) and Lemma 6 we obtain

$$
\begin{aligned}
T(r, F) \leq & T\left(r, F_{1}\right)+S(r, F) \\
\leq & N_{2}\left(r, 0 ; F_{1}\right)+N_{2}\left(r, 0 ; G_{1}\right)+3 \bar{N}\left(r, \infty ; F_{1}\right)+2 \bar{N}\left(r, \infty ; G_{1}\right) \\
& +\bar{N}_{*}\left(r, \infty ; F_{1}, G_{1}\right)+2 \bar{N}\left(r, 0 ; F_{1}\right)+\bar{N}\left(r, 0 ; G_{1}\right) \\
& +S\left(r, F_{1}\right)+S\left(r, G_{1}\right) \\
\leq & N_{2}(r, 0 ; F)+N_{2}(r, 0 ; G)+3 \bar{N}(r, \infty ; F)+2 \bar{N}(r, \infty ; G) \\
& +\bar{N}_{*}(r, \infty ; F, G)+2 \bar{N}(r, 0 ; F)+\bar{N}(r, 0 ; G)+S(r, F)+S(r, G) \\
\leq & N_{2}(r, 0 ; F)+N_{2}(r, 0 ; G)+4 \bar{N}(r, \infty ; F)+2 \bar{N}(r, \infty ; G) \\
& +2 \bar{N}(r, 0 ; F)+\bar{N}(r, 0 ; G)+S(r, F)+S(r, G) .
\end{aligned}
$$


Using (15), Lemma 2 and Lemma 3 we obtain from (8)

$$
\begin{aligned}
& (n-1) T(r, f) \leq N_{2}(r, 0 ; F)+N_{2}(r, 0 ; G)+4 \bar{N}(r, \infty ; F)+2 \bar{N}(r, \infty ; G) \\
& +2 \bar{N}(r, 0 ; F)+\bar{N}(r, 0 ; G)-N(r, \infty ; f)-N\left(r, 0 ; f^{(k)}\right) \\
& +S(r, f)+S(r, g) \\
& \leq 4 \overline{\mathrm{N}}(r, 0 ; f)+3 \overline{\mathrm{N}}(\mathrm{r}, 0 ; g)+\mathrm{N}_{2}\left(\mathrm{r}, 0 ; f^{(\mathrm{k})}\right)+\mathrm{N}_{2}\left(\mathrm{r}, 0 ; \mathrm{g}^{(\mathrm{k})}\right) \\
& +4 \bar{N}(r, \infty ; f)+2 \bar{N}(r, \infty ; g)+2 \bar{N}\left(r, 0 ; f^{(k)}\right)+\bar{N}\left(r, 0 ; g^{(k)}\right) \\
& -N(r, \infty ; f)-N\left(r, 0 ; f^{(k)}\right)+S(r, f)+S(r, g) \\
& \leq 4 \bar{N}(r, 0 ; f)+3 \bar{N}(r, 0 ; g)+N\left(r, 0 ; g^{(k)}\right)+2 \bar{N}\left(r, 0 ; f^{(k)}\right) \\
& +\overline{\mathrm{N}}\left(\mathrm{r}, 0 ; \mathrm{g}^{(\mathrm{k})}\right)+3 \mathrm{~N}(\mathrm{r}, \infty ; f)+2 \overline{\mathrm{N}}(\mathrm{r}, \infty ; \mathrm{g}) \\
& +\mathrm{S}(\mathrm{r}, \mathrm{f})+\mathrm{S}(\mathrm{r}, \mathrm{g}) \\
& \leq 4 \overline{\mathrm{N}}(\mathrm{r}, 0 ; f)+3 \overline{\mathrm{N}}(\mathrm{r}, 0 ; g)+\mathrm{N}(\mathrm{r}, 0 ; g)+2 \mathrm{~N}_{\mathrm{k}+1}(\mathrm{r}, 0 ; f) \\
& +N_{k+1}(r, 0 ; g)+(2 k+3) N(r, \infty ; f)+(2 k+2) \bar{N}(r, \infty ; g) \\
& +\mathrm{S}(\mathrm{r}, \mathrm{f})+\mathrm{S}(\mathrm{r}, \mathrm{g}) \\
& \leq(2 k+6) \bar{N}(r, 0 ; f)+(k+4) \bar{N}(r, 0 ; g)+N(r, 0 ; g) \\
& +(2 k+3) \mathrm{N}(r, \infty ; f)+(2 k+2) \bar{N}(r, \infty ; g) \\
& +\mathrm{S}(\mathrm{r}, \mathrm{f})+\mathrm{S}(\mathrm{r}, \mathrm{g}) \\
& \leq \frac{k+4}{k}(\mathrm{~N}(r, 0 ; f)+\mathrm{N}(r, 0 ; g))+\frac{k+2}{k} \mathrm{~N}(r, 0 ; f) \\
& +N(r, 0 ; g)+(2 k+3) N(r, \infty ; f)+(2 k+2) \bar{N}(r, \infty ; g) \\
& +S(r, f)+S(r, g) \\
& \leq\left(1+\frac{4}{k}\right)(T(r, f)+T(r, g))+\left(2 k+\frac{2}{k}+4\right) T(r, f) \\
& +(2 k+3) T(r, g)+S(r, f)+S(r, g) \text {. }
\end{aligned}
$$

This gives

$$
\begin{aligned}
\left(n-2 k-\frac{2}{k}-5\right) T(r, f) \leq & \left(1+\frac{4}{k}\right)(T(r, f)+T(r, g)) \\
& +(2 k+3) T(r, g)+S(r, f)+S(r, g) .
\end{aligned}
$$

Similarly,

$$
\begin{aligned}
\left(n-2 k-\frac{2}{k}-5\right) T(r, g) \leq & \left(1+\frac{4}{k}\right)(T(r, f)+T(r, g)) \\
& +(2 k+3) T(r, f)+S(r, f)+S(r, g) .
\end{aligned}
$$


In view of (16) and (17) we obtain

$$
\left(n-4 k-\frac{10}{k}-10\right)(T(r, f)+T(r, g)) \leq S(r, f)+S(r, g),
$$

which contradicts our assumption that $n>4 k+10 / k+10$.

Case 2 We now assume that $\mathrm{H}=0$. That is

$$
\left(\frac{F_{1}^{\prime \prime}}{F_{1}^{\prime}}-\frac{2 F_{1}^{\prime}}{F_{1}-1}\right)-\left(\frac{G_{1}^{\prime \prime}}{G_{1}^{\prime}}-\frac{2 G_{1}^{\prime}}{G_{1}-1}\right)=0 .
$$

Integrating both sides of the above equality twice we get

$$
\frac{1}{\mathrm{~F}_{1}-1}=\frac{A}{\mathrm{G}_{1}-1}+\mathrm{B}
$$

where $\mathrm{A}(\neq 0)$ and $\mathrm{B}$ are constants. From (18) it is clear that $\mathrm{F}_{1}$ and $\mathrm{G}_{1}$ share $1 C M$ and hence they share the value 1 with weight 2, and therefore, $\mathrm{n}>\mathrm{k}+4 / \mathrm{k}+4$. Now we consider the following three subcases.

Subcase 4 Let $\mathrm{B} \neq 0$ and $\mathrm{A}=\mathrm{B}$. Then from (18) we get

$$
\frac{1}{F_{1}-1}=\frac{B G_{1}}{G_{1}-1} \text {. }
$$

If $\mathrm{B}=-1$, then from (19) we obtain

$$
\mathrm{F}_{1} \mathrm{G}_{1}=1
$$

i.e.,

$$
f^{n} f^{(k)} g^{n} g^{(k)}=z^{2} \text {. }
$$

Therefore by Lemma 7 we obtain $\mathrm{f}(z)=\mathrm{c}_{1} e^{\mathrm{c} z^{2}}, \mathrm{~g}(z)=\mathrm{c}_{2} e^{-\mathrm{c} z^{2}}$, where $\mathrm{c}_{1}, \mathrm{c}_{2}$ and $\mathrm{c}$ are three constants satisfying $4\left(\mathrm{c}_{1} \mathrm{c}_{2}\right)^{\mathrm{n}+1} \mathrm{c}^{2}=-1$. If $\mathrm{B} \neq-1$, from (19) we have $\frac{1}{\mathrm{~F}_{1}}=\frac{\mathrm{BG}_{1}}{(1+\mathrm{B}) \mathrm{G}_{1}-1}$, and therefore, $\overline{\mathrm{N}}\left(\mathrm{r}, \frac{1}{1+\mathrm{B}} ; \mathrm{G}_{1}\right)=\overline{\mathrm{N}}\left(\mathrm{r}, 0 ; \mathrm{F}_{1}\right)$. Now using the second fundamental theorem of Nevanlinna, we get

$$
\begin{aligned}
& \mathrm{T}(\mathrm{r}, \mathrm{G}) \leq \mathrm{T}\left(\mathrm{r}, \mathrm{G}_{1}\right)+\mathrm{S}(\mathrm{r}, \mathrm{G}) \\
& \leq \bar{N}\left(r, 0 ; G_{1}\right)+\bar{N}\left(r, \frac{1}{1+B} ; G_{1}\right)+\bar{N}\left(r, \infty ; G_{1}\right)+S(r, G) \\
& \leq \overline{\mathrm{N}}\left(\mathrm{r}, 0 ; \mathrm{F}_{1}\right)+\overline{\mathrm{N}}\left(\mathrm{r}, 0 ; \mathrm{G}_{1}\right)+\overline{\mathrm{N}}\left(\mathrm{r}, \infty ; \mathrm{G}_{1}\right)+\mathrm{S}(\mathrm{r}, \mathrm{G}) \\
& \leq \bar{N}(r, 0 ; F)+\bar{N}(r, 0 ; G)+\bar{N}(r, \infty ; G)+S(r, G) \text {. }
\end{aligned}
$$


Using (20), Lemma 2 and Lemma 3 we obtain from (9)

$$
\begin{aligned}
(n-1) T(r, g) \leq & \bar{N}(r, 0 ; F)+\bar{N}(r, 0 ; G)+\bar{N}(r, \infty ; G)-N(r, \infty ; g) \\
& -N\left(r, 0 ; g^{(k)}\right)+S(r, g) \\
\leq & \bar{N}(r, 0 ; f)+\bar{N}(r, 0 ; g)+\bar{N}(r, \infty ; g)+\bar{N}\left(r, 0 ; f^{(k)}\right) \\
& +\bar{N}\left(r, 0 ; g^{(k)}\right)-N(r, \infty ; g)-N\left(r, 0 ; g^{(k)}\right) \\
& +S(r, f)+S(r, g) \\
\leq & \bar{N}(r, 0 ; f)+\bar{N}(r, 0 ; g)+N_{k+1}(r, 0 ; f)+k \bar{N}(r, \infty ; f) \\
& +S(r, f)+S(r, g) \\
\leq & \frac{k+2}{k} N(r, 0 ; f)+\frac{1}{k} N(r, 0 ; g)+k \bar{N}(r, \infty ; f) \\
& +S(r, f)+S(r, g) \\
\leq & \frac{1}{k}(T(r, f)+T(r, g))+\left(k+\frac{1}{k}+1\right) T(r, f) \\
& +S(r, f)+S(r, g) .
\end{aligned}
$$

Thus we obtain

$$
\left(n-k-\frac{3}{k}-2\right)(T(r, f)+T(r, g)) \leq S(r, f)+S(r, g)
$$

a contradiction as $\mathrm{n}>\mathrm{k}+4 / \mathrm{k}+4$.

Subcase 5 Let $\mathrm{B} \neq 0$ and $\mathrm{A} \neq \mathrm{B}$. Then from (18) we get $\mathrm{F}_{1}=\frac{(\mathrm{B}+1) \mathrm{G}_{1}-(\mathrm{B}-\mathrm{A}+1)}{\mathrm{BG}_{1}+(\mathrm{A}-\mathrm{B})}$, and so, $\overline{\mathrm{N}}\left(\mathrm{r}, \frac{\mathrm{B}-\mathrm{A}+1}{\mathrm{~B}+1} ; \mathrm{G}_{1}\right)=\overline{\mathrm{N}}\left(\mathrm{r}, 0 ; \mathrm{F}_{1}\right)$. Proceeding as in Subcase 4 we obtain a contradiction.

Subcase 6 Let $B=0$ and $A \neq 0$. Then from (18) we get $F_{1}=\frac{G_{1}+A-1}{A}$ and $\mathrm{G}_{1}=A \mathrm{~F}_{1}-(\mathrm{A}-1)$. If $\mathrm{A} \neq 1$, we have $\overline{\mathrm{N}}\left(\mathrm{r}, \frac{\mathrm{A}-1}{\mathrm{~A}} ; \mathrm{F}_{1}\right)=\overline{\mathrm{N}}\left(\mathrm{r}, 0 ; \mathrm{G}_{1}\right)$ and $\bar{N}\left(r, 1-A ; G_{1}\right)=\bar{N}\left(r, 0 ; F_{1}\right)$. Using the similar arguments as in Subcase 4 we obtain a contradiction. Thus $\mathrm{A}=1$ which implies $\mathrm{F}_{1}=\mathrm{G}_{1}$, and therefore, $f^{n} f^{(k)}=g^{n} g^{(k)}$.

This completes the proof of Theorem 1.

Proof of Theorem 2. Using Lemma 8 and proceeding similarly as in the proof of Theorem 1, we can prove Theorem 2.

\section{Acknowledgements}

The author would like to thank the referee for his/her valuable suggestions and comments towards the improvement of the paper. 


\section{References}

[1] T. C. Alzahary, H. X. Yi, Weighted value sharing and a question of I. Lahiri, Complex Var. Theory Appl., 49 (2004), 1063-1078.

[2] A. Banerjee, Uniqueness of meromorphic functions sharing two sets with finite weight, Port. Math. (N.S.) 65 (2008), 81-93.

[3] W. Bergweiler, A. Eremenko, On the singularities of the inverse to a meromorphic function of finite order, Rev. Mat. Iberoame., 11 (1995), $355-373$.

[4] Y. H. Cao, X. B. Zhang, Uniqueness of meromorphic functions sharing two values, J. Inequal. Appl., 2012:100.

[5] H. H. Chen, M. L. Fang, The value distribution of $\mathrm{f}^{n} \mathrm{f}^{\prime}$, Sci. China Math., 38 (1995), 789-798.

[6] M. L. Fang, A note on a problem of Hayman, Analysis (Munich), 20 (2000), 45-49.

[7] M. L. Fan, H. L. Qiu, Meromorphic functions that share fixed points, J. Math. Anal. Appl., 268 (2002), 426-439.

[8] W. K. Hayman, Meromorphic functions, The Clarendon Press, Oxford (1964).

[9] I. Lahiri, Weighted sharing and uniqueness of meromorphic functions, Nagoya Math. J., 161 (2001), 193-206.

[10] I. Lahiri, Weighted value sharing and uniqueness of meromorphic functions, Complex Var. Theory Appl., 46 (2001), 241-253.

[11] P. Sahoo, Meromorphic functions that share fixed points with finite weights, Bull. Math. Anal. Appl., 2 (2010), 106-118.

[12] J. F. Xu, Z. L. Zhang, Some inequalities of differential polynomials, Math. Inequal. Appl., 12 (2009), 99-113.

[13] C. C. Yang, On deficiencies of differential polynomials II, Math. Z., 125 (1972), 107-112.

[14] C. C. Yang, X. H. Hua, Uniqueness and value sharing of meromorphic functions, Ann. Acad. Sci. Fenn. Math. 22 (1997), 395-406. 
[15] L. Yang, Value distribution theory, Springer- Verlag, Berlin, 1993.

[16] H. X. Yi, C. C. Yang, Uniqueness theory of meromorphic functions, Science Press, Beijing, 1995.

[17] L. Zalcman, On some problems of Hayman, Preprint (Bar-Ilan University), 1995.

[18] J. L. Zhang, L. Z. Yang, Some results related to a conjecture of R. Bruck, J. Inequal. Pure Appl. Math., 8 (2007), Art. 18.

Received: 18 October 2012 